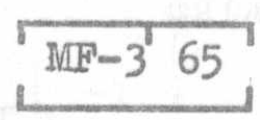

$\Gamma$
MILITARY TECHNICAL COLLEGE

$$
\text { CAIRO - EGYPT }
$$

$1-$

2-

$3-$

\title{
ASSESSMENT OF ERFB-BB PROJECTILE
}

HASSAN YAKOUT ${ }^{*}$ and MOHAMED S. ABDEL-KADER ${ }^{*}$

\section{ABSTRACT}

The development of extended $r$ ange rounds has followed a clearly traceable line of evolution starting with the Racket-Assisted Projectiles (RAP), passing by Extended Range Sub-Calibre (ERSC) projectiles and Extended Range Sub-Bore (ERSB) projectiles and ending with Extended Range Full Bore projectiles with Boat Tail (ERFB-BT) or Base Bleed (ERFB-BB). The most interesting of all the extended range projectiles developed so far is undoubtedly the very long range ERFB-BB ones, which incorporate the best ballistic features of the other extended range projectiles and achieve the maximum possible payload capacity.

In this treatise, a historical review of the development of highexplosive, extended range projectilles is first furnished. The principle of base bleed is then discussed and its use in modern projectiles illustrated via a newly designed ERFB-BB projectile. Necessary calculations of range, accuracy and lethality are performed. In doing so, different base drag reduction ratios are considered. Effects of delayed ignition and slight variations in time of burning of the base bleed charge on range are also studied, and maximum $r$ ange is found to be considerably sensitive to these variations. Possibility of local production of the proposed design is investigated. Anticipated technical difficulties are singled out and possible solutions proposed.
\end{abstract} College, Cairo, EGYPT. 
Today's tactics demand that artillery units be positioned at greater depth behind the front line to insure survivability. Modern battlefield deployment scenarios, with fluid fromts and improved enemy firepower, require longer ranges of friendly fire support units. Extended range is most significant in terms of area coverage. The coverage allaws the artillery unit to engage targets earlier, fire on more targets, and provide greater flank coverage. Greater range also necessitates fewer displacements for the atrillery units.

Range extension of conventional munition can be achieved by virtue of different solution strategies [1]. These include: (i) increasing projectile muzzle velocity, (ii) improving its ballistic performance, and (iii) designing rocket-assisted projectiles. Each of these strategies has its own advantages and limitations, and optimized procedures that take into account a number of tactical and economical considerations are usually undertaken to come up with 'optimal' designs.

The development of Extended Range (ER) ammunition has followed a clearly traceable line of evolution since the early 1970's when the United States of America has adopted the Rocket-Assisted Projectile (RAP) solution strategy for range extension. Figure 1 depicts one such design, aduanced by BPD of Italy for a series of the $155 \mathrm{~mm}$ howitzers. RAPs have two fundamental functional drawbacks, however; the space occupied by the rocket motor reduces the High Explosive (HE) capacity, and accordingly the effect of the projectile by about $30 \%$ and the in-flight rocket boost adds more elements of dispersion to the ballistic trajectory, thus reducing accuracy.

A different, fully ballistic approach consists in streamlining the conventional projectile in order to limit its aerodynamic wave drag. This can be achieved by eliminating the cylindrical center section and incorporating a long, full-ogive body, tapering all the way from the driving band at the base to the nose fuze. This reduces aerodynamic wave drag by about $30 \%$ and simultaneously improves flight stability. In addition, the frontal area (equivalently the diameter) and the mass can be reduced, Which respectively produce a further drag reduction and raise muzzle velocity.

The resulting Extended Range Sub-Calibre (ERSC) projectile requires two sabots to insure regularity of projectile motion inside weapon barrel (the rear sabot carrying the driving band), both of which are to be discarded in flight. One such projectile was produced by Space Research Corporation (SRC) that provided a range of more than $22 \mathrm{Km}$ for the $155 \mathrm{~mm}$, 23-calibre-barrelled M109 self-propelled howitzer, compared to the maximum $14.6 \mathrm{Km}$ of the standard M107 projectiles (i.e. a range extension of about $50 \%$ [2]. However, the ERSCs reduce weight and payload comporised lethality so that it could only be used as special purpose ammunition.

Lo arercone the deficiencies of the ERSL frojeciles, an Extended 
C

Range Sub-Bore (ERSB) projectile, with a nearly full calibre (bore) projectile was developed. This enabled the front sabot of the ERSC to be replaced by four aerodynamically streamlined nubs that provide support at the mid section. A discarding, nonmetallic driving band provided the necessary support at the base and transmitted the rotational force. A tapering boat tail was also added behind the driving band to reduce the wake zone behind the shell base, thus cutting down the amount of base drag. Lethality of the ERSB projectile is close to that of the standard projectile.

Difficulties in developing the discarding driving band of the ERSB and its still slightly reduced lethality, shifts emphasis towards Extended Range Full Bore (ERFB) projectiles, which incorporate the best features of the ERSBs, together with the maximum possible payload capacity. The fixed nubs of the early version of the ERFBs were welded on, but those of the new series are machined from the forged body, thus reducing cost, enhancing productivity, and raising reliability. The latest version of the ERFBs achieves a maximum $r$ ange in excess of $26 \mathrm{Km}$ with the 39-calibre barrel of the American M198 towed howitzer, $8 \mathrm{Km}$ (or about $45 \%$ ) more than the M107 projectile fired from the same howitzer.

The ERFB's low drag aerodynamic profile reduces flight time, makes the trajectory therefore less susceptible to meteorological perturbations and, in consequence, improves precision. Its hollow base reduces transverse momoent of inertia and shifts centre of gravity forward, thus improving the stability of the projectile.

The most interesting of all the ERFB projectiles developed so far is undoubtedly the very long range, fourth-generation ERFB Base Bleed (or Base Burn) (ERFB-BB) projectile. It is actually a normal ERFB projectile, but with a different boattail containing the base bleed unit. Although it has a lower muzzle velocity, it Provides an increase in maximum $r$ ange of about 15 to $30 \%$ depending on the weapon. Figure 2 exhibits the first three generations of the $155 \mathrm{~mm}$ ER projectiles, together with the standard M107 HE projectile, whilst Fig.3 shows the fourth generation, i.e. ERFB$B B$ projectle, along with the components of the base burn unit. Moreover, Table 1 shows the maximum $r$ ange of $s$ tandard, RAP, ERFB and ERFB-BB projectiles for a number of $155 \mathrm{~mm}$ weapons [3]. In the remainder of this work, more 1 ight will be shed on the ERFBBB projectile, primarily because of its salient features.

\section{BASIC CONCEPTS}

During its flight, the projectile is acted upon by a number of forces. These include the gravitational force, air resistance and Coriolis force accounting for earth rotation. The first one is normally assumed to be constant in exterior ballistic calculations of artillery projectiles, whilst the third one is customarily not considered, because of its negligible effect.

The air resistance force $R$ can be decomposed into three components ( (i) head resistance $R_{H R}$, (ii) skin friction $R_{S F}$ and (iii) L 
The head resistance experienced by the projectile is caused by compression waves generated ahead of the projectile path due to its motion in air. The magnitude of this component increases rapidly in the supersonic region due to the formation of shock waves at the projectile nose. The skin friction is caued by shear stress developed due to the air viscous effect on the projectile wet area. Its effect on projectile motion, however, is relati-

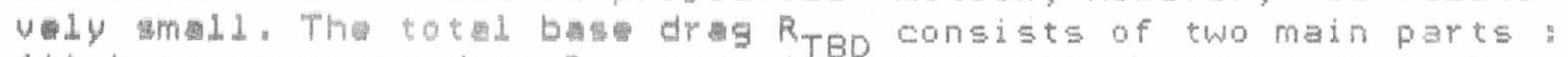
(i) base preasure drag $R_{B D}$ and (ii) boattail pressure drag $R_{B T}$ The base pressure dragis caused by the formation of wake just behind the projectile tail. This region is characterized by low pressure distribution deviated considerably from that of the upstream conditions, and attributed to the inability of air streamlines to follow the projectile profile. On the other hand, the boattail pressure drag results from the pressure gradient developed along the boattail surface.

Drag prediction represents a major part of the calculation of projectile trajectory, which is the main task of Exterior Ballistics. A common practice is to estimate drag empirically using air resistance 1 aws, such as siacci, 1930 and 1943. These laws express air resistance in the general form:

$$
R=P_{d} S C_{x}(M)
$$

Where $p_{d}=1 / 2 \rho v^{2}$ is the dynamic pressure, $\rho$ is the air specific mass, $v^{d}$ is the projectile velocity, s is the projectile crosssectional area, $C,(M)=i C_{x}^{*}(M)$ is the total drag coefficients $M=$ $u / a$ is the Mach number, and a is air sonic velocity. The function $C_{x}^{*}(M)$ is the drag coefficient of some standard projectile, which i determined experimentally and varies from one law to another, and $i$ is a constant known as the projectile shape coefficient. It is thus implied that air resistance laws assume that the function $C_{x}(M)$ has the same form of the chosen 1 aw of air resistance and differs only by a constant multiplier i. This multiplier is a characteristic of each projectile and is normally determined either by actual firing or in wind tunnels. Experimental work indicates that this multiplier depends only on the projectile firing angle.

Equations (1) and (2) suggest that the total air resistance be written in the alternative form:

$$
R=P_{d} S\left(C_{H R}+C_{S F}+C_{T B D}\right)
$$

where $\mathrm{C}_{\text {HR }} i \mathrm{~s}$ the head drag coefficient, $\mathrm{C}_{\mathrm{SF}}$ is the surface friction drag coefficient and C TBD is the total base drag coefficient, which can be decomposeg into a base drag component $\mathrm{C}_{\mathrm{BD}}$ and a boattail one $\mathrm{C}_{\mathrm{BT}}$, that is:

$$
\mathrm{C}_{\text {TBD }}=\mathrm{C}_{\mathrm{BD}}+\mathrm{C}_{\mathrm{BT}} \text { * }
$$

Figure 4 showe the total drag coefficient for a tupical HE shell based on Siacci's law, together with its components of Eqs. (3) Land (4). It is clear from this figure that the total base drag 
coefficient represents about $75 \%$ of the total drag coefficient at subsonic velocities, about $60 \%$ at transonic velocities and about $50 \%$ in supersonic region [4]. That is probably why research activity concerning $r$ ange extension has been mostly concerned with reducing base drag, particularly via base bleeding.

\section{BASE BLEED PRINCIPLE}

Firing projectiles with tracers indicates that mass flow out of the base reduces base drag. Wind tunnel tests have also indicated that base drag can be considerably reduced if certain conditions are established for gases flowing out of the base plane. The mass flow rate, for instance, should not exceed certain magnitude, nor should burning take place far from the base plane. Resulting thrust must have negligible effect in relation to the forces due to pressure increase on the base plane. In fact, it has been reported that by burning hydragen close to the base plane, base drag has been completely diminished [5].

In base bleed projectiles, a base bleed unit is usually employed, Which generates hot gases that are expelled in to the wake region. Gas flow through the exhaust port is subsonic and provides negligible thrust. However, it increases base pressure and reduces base drag. The amount of reduction depends on the firing and flight conditions, but typically varies between 60 and $80 \%$. There are a number of practical problems, which arise when designing a projectile with a base bleed unit. Large acceleration of projectile during its motion down weapon barrel, the relatively large volume of the unit, type of base bleed charge and its burning characteristics represent some of these problems.

The base bleed charge is ignited by the gases of the main charge inside gun barrel, but due to the sudden pressure drop at the muzzle, it is important to have sustaining igniter to eliminate the possibility of coughing of the base bleed unit. This igniter is also initiated by the propellant gases and burns for about 1 to $1.5 \mathrm{sec}$, whereas the base bleed charge continues to burn for a time of about $1 / 3$ of the maximum flight time, resulting in $a$ range extension of up to $25 \%$. Longer bleeding times cause slight increase in range and require larger bleeding units, which affects the lethality of the projectile adversely $[1,5]$.

To further ascertain these arguments, the trajectory of the 155 $\mathrm{mm} H E$ shell with a base bleed time, $T_{B}$, ranging from 0.2 to 0.6 of the flight time at maximum range of the original shell, $T$, has been predicted, as shown in Fig.5. In doing so, it has Been assumed that the total drag coefficient was reduced by $40 \%$ due to bleeding (correspondint to a reduction of about $80 \%$ in base drag). It is clear from this figure that the maximum range, $X$, increases with increasing $T_{B}$. Plotting $X$ with $T_{B}$ in Fig.6, however, shows that for $T_{B}$ greater than $0.4 T_{5}$, only slight increase in $X$ is achieved.

\section{PROPOSED DESIGN AND DISCUSSIONS}

A number of $155 \mathrm{~mm}$ high explosive, base bleed projectiles have Leen recently designed by international armament firms, such asj 
$\Gamma$.

BPD (Italy) and SRC (USA), which are shown in Fig.3. A similar version of the standard $155 \mathrm{~mm}$ high explosive projectile with base bleed for use with M109 Al howitzer is proposed herein. In this proposal, both shape and dynamic characteristics of the shell were kept as close as possible to those of the original projectile. Theoretical external ballistic calculations of the trajectory of the $155 \mathrm{~mm}$ HE, base bleed projectile proposed herein show that a range exterision of about $22 \%$ can be achieved, assuming a reduction of $80 \%$ in base drag due to bleeding. If the base drag is entirely eliminated, hypothetically, range can be further extended to $23.1 \mathrm{Km}$ (about $28 \%$ extension). Corresponding trajectories are shown in Fig.7. Further range extensian could have been achieved, if the projectile shape had been strea$\mathrm{mlined}$ and its length increased. In such a case, the ballistic coefficient needs to be determined, essentially via a series of laboratory and field tests.

To study the effect of irrigularity of burning of the base bleed charge, the time of burning $T_{B}=0.4 \mathrm{~T}_{5}$ was allowed to change to 'ugriations in maximum Pange were predicted, and are shown in Fig. 8. It is clear that considerable variations in range are obtained $(\Delta X / X=-2.43: 1.98 \%$ for fully reduced base drag), which increase with base $d r a g$ reduction ratio. This explains why actual firing tests of base bleed projectiles may give unsatisfactory dispersion patterns. Figure 9 illustrates the effect of slight variations in time of ignition and confirms the above arguments.

Predictions of range at different elevation angles for the proposed base bleed projectile with different base drag reduction ratios are depicated in Fig.10. Corresponding flight times and maximum altitudes are respectively shown in Figs? 11 and 12 versus range. Corresponding values for the standard projectile are also included in the three figures for comparison. It is evident that the base bleed projectile reaches longer $r$ anges at all elevation angles. It can also cover any range in a shorter time than the standard projectile. Furthermore, the trajectory summit of the base bleed projectile is reached at longer horizontal distance and flight time. This trend proceeds with increasing base drag reduction, in consistence with previous findings.

Because the bursting charge is reduced by about $10 \%$ in the current design, both fragmentation and deriolition effects of projectile on target are also reduced. The effect can be improved, however, using a bursting charge with better performance. Stability of the projectile during flight has been checked and was found satisfactory. Real field tests are needed to assess accuracy and consistency.

From a production standpoint, the proposed projectile body can be produced by hot pressing without major difficulties. In this case, however, pressing involves creation of two cavities, a greater one for the bursting charge and a smaller one for the base bleed unit. Design, production and performance of the base bleed composition represent the main difficulty in bringing this proposal into reality. Hard team work involving a relatively [great number of laboratory and field tests is expected and suc-j 
r cess is anticipated. The sustaining igniter represents a major
part of the aforementioned work. Preparation of firing tables for
. Pork. No the proposed projectile is another area of extensive work. No major difficulties are anticipated in this regard.

\section{CONCLUSION}

The $155 \mathrm{~mm}$ high explosive projectile with base bleed proposed herein can reach a theoretical maximum $r$ ange of about $22 \mathrm{Km}$, thus achieving a range extension of approximately $22 \%$ in comparison with the original high explosive projectile. This range is essentially due to base drag reduction of $80 \%$. On the other hand, a slight reduction in projectile effect on target is the tradeoff. Irregularity of ignition and subsequent burning of base bleed charge is shown to affect accuracy of fire diversely.

\section{REFERENCES}

1.Yakout,H. and Abdel-kader,M.S.,"Range Extension Via Base Bleeding", AME 4th Conf., M.T.C., Cairo (1990).

2.Furlong,R.D.M., "Extended Range Full Bore and Base Bleed 155 mm Ammunition", Int. Def. Review, 6, 755-760 (1982).

3.Anon," Can Your $155 \mathrm{~mm}$ Artillery Exceed the $20 \mathrm{Km}$ Range ?", Into Def. Review, 17, 1773 (1984).

4.El-Ashmawy,A.R.," "Drag Prediction Methodology for Missiles at Supersonic Speeds ", M.Sc. Thesis, M.T.C., Cairo (1986).

5. Andersson, K., Gunners,N.E. and Hellgren, R.," "Swedish Base Bleed - Increasing the Range of Artillery Projectiles Through Base Flow", Res. Inst. National Defence, Sweden (1976).

Table 1. Maximum $r$ ange achieved (in $\mathrm{Km}$ ) by weapons and by projectile/payload.

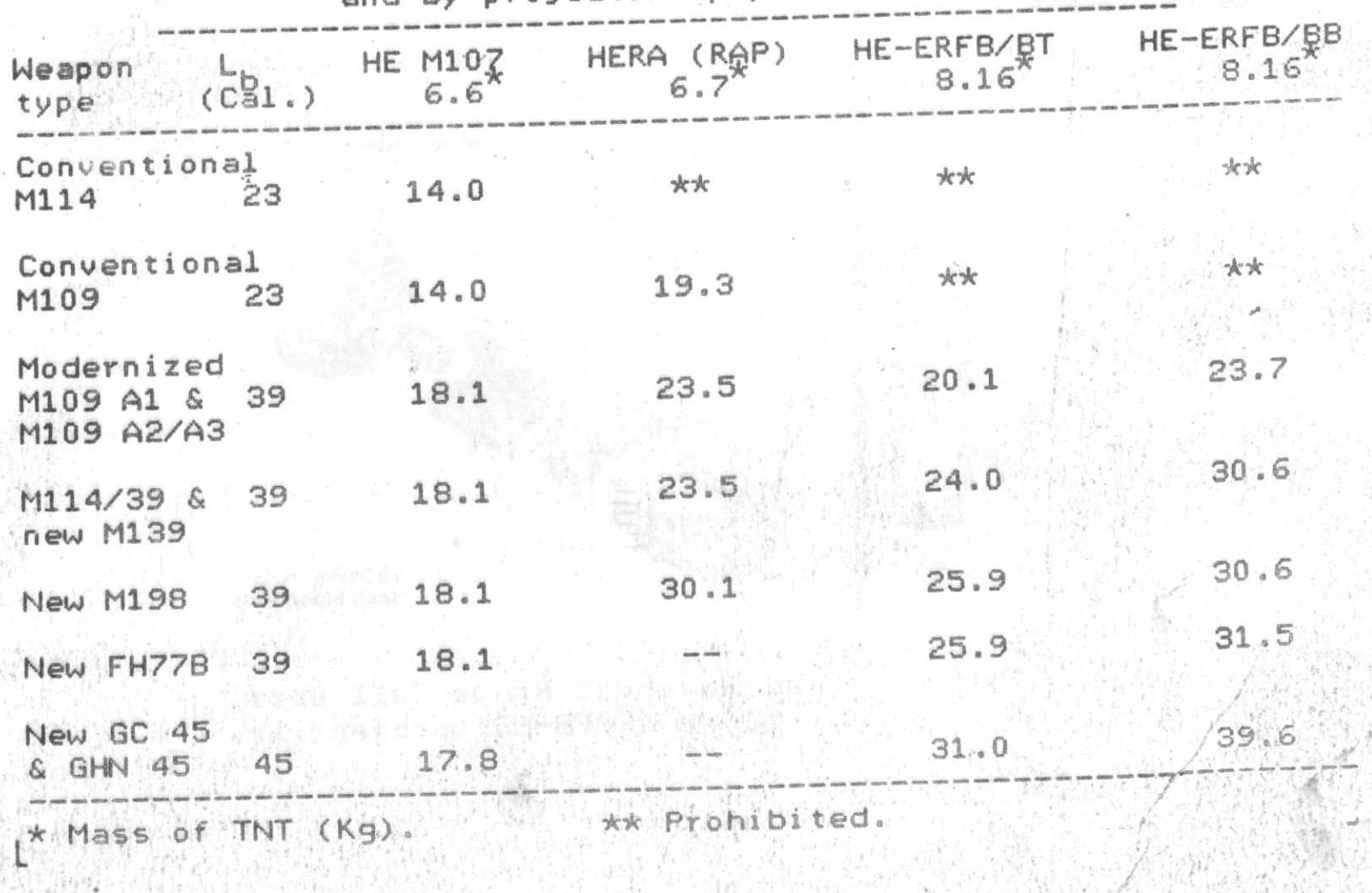




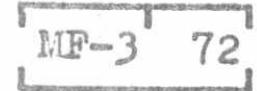

$\Gamma$

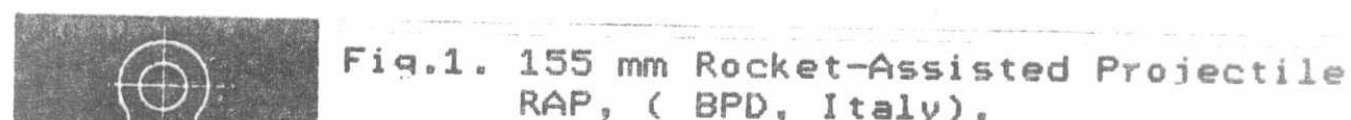
RAP, ( BPD, Italy).

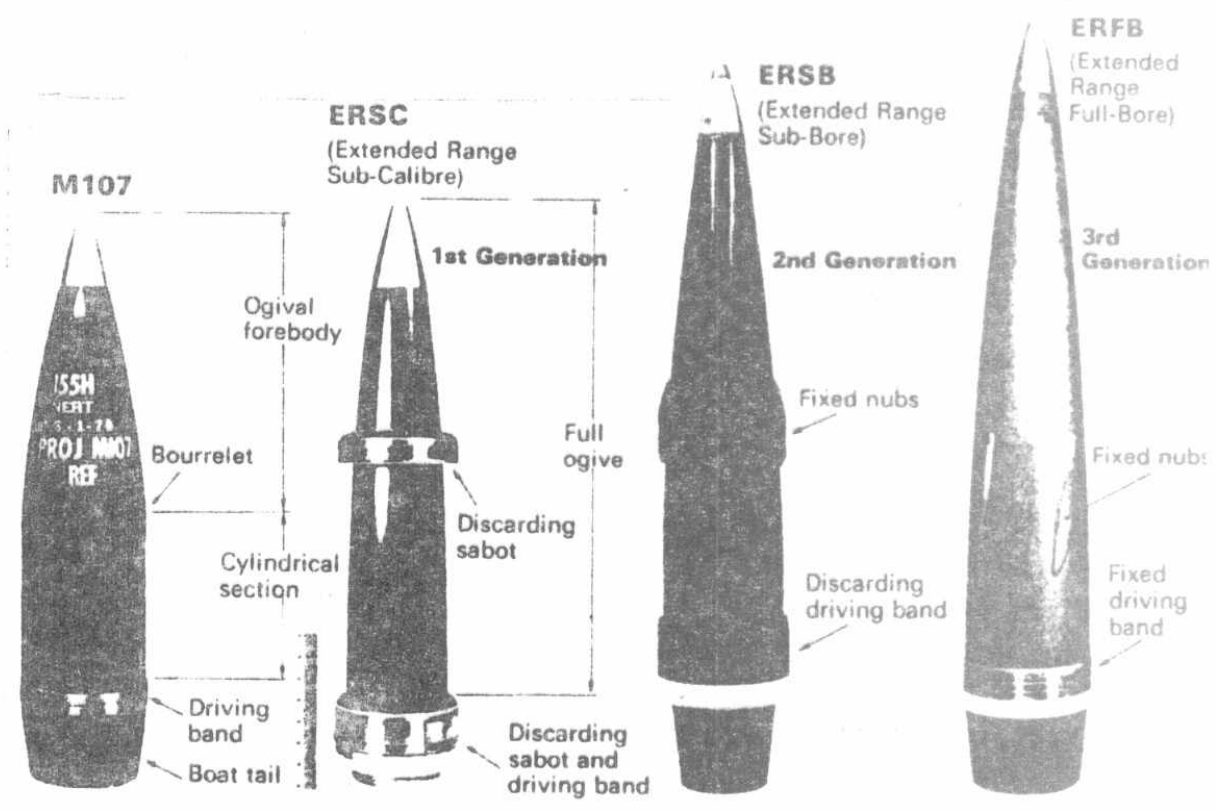

Fig.2. 15Emm standard and extended range ammunition
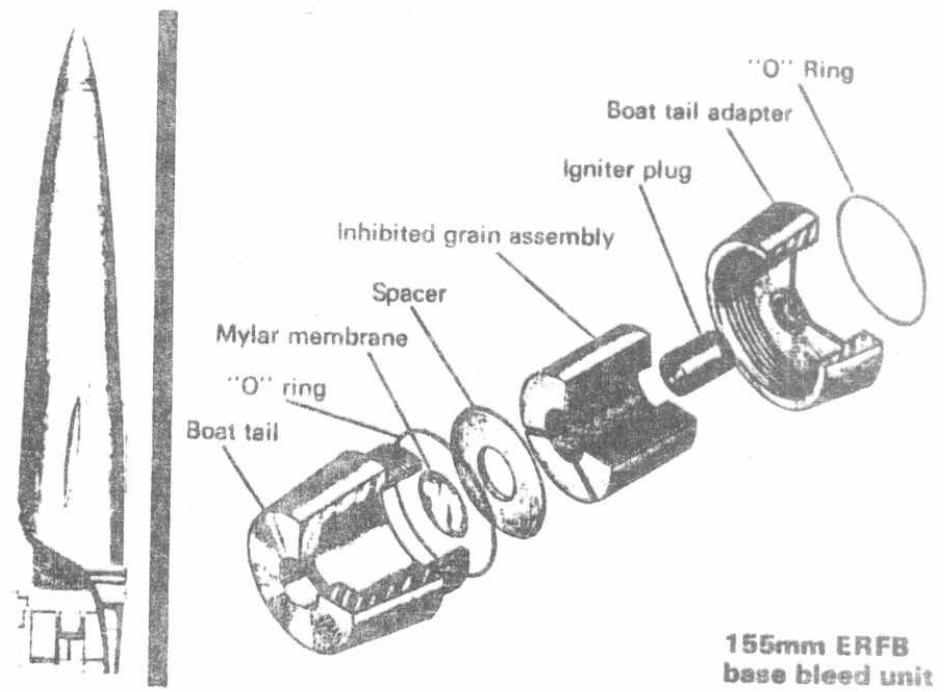

Fiq.3. $155 \mathrm{~mm}$ Extended Range Full Bore Base Bleed (ERFB-BB) projectile. 


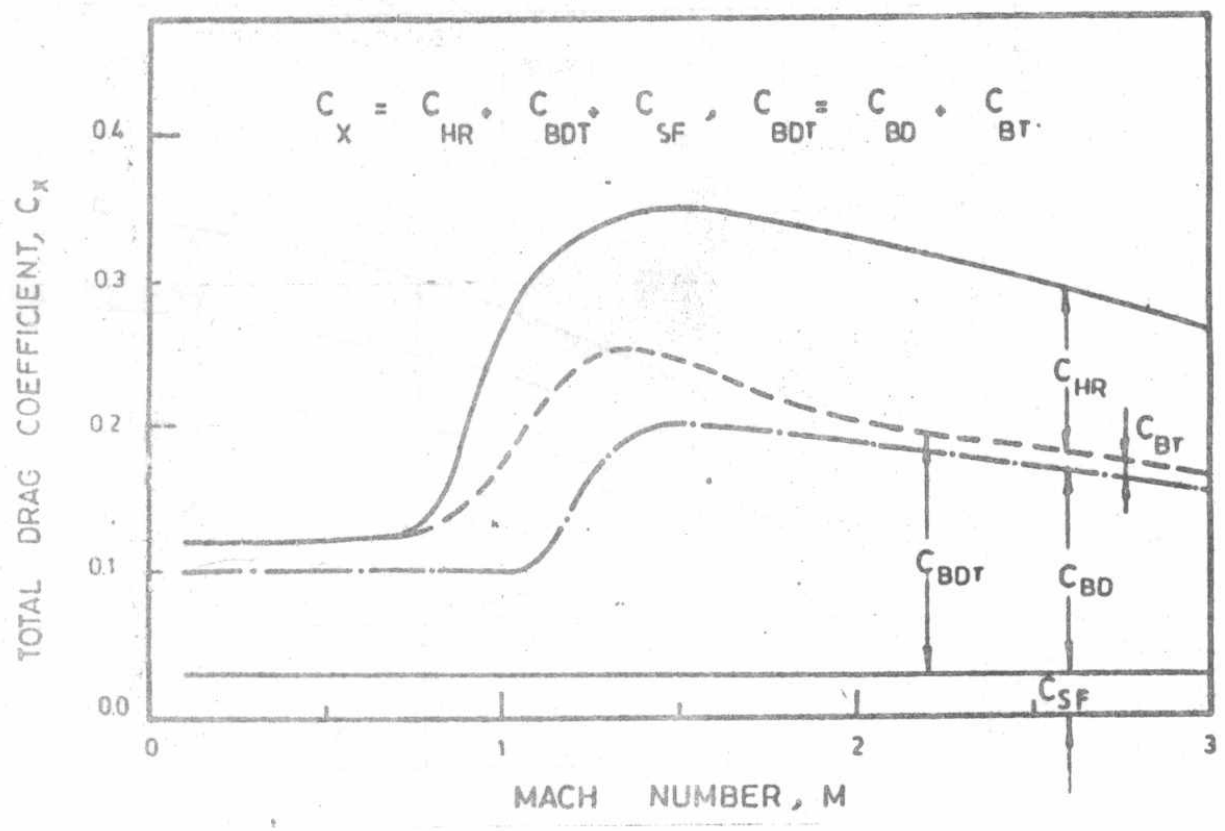

Fig.4. Siacci's total drag coefficient verses Mach number (for typical HE shel1).

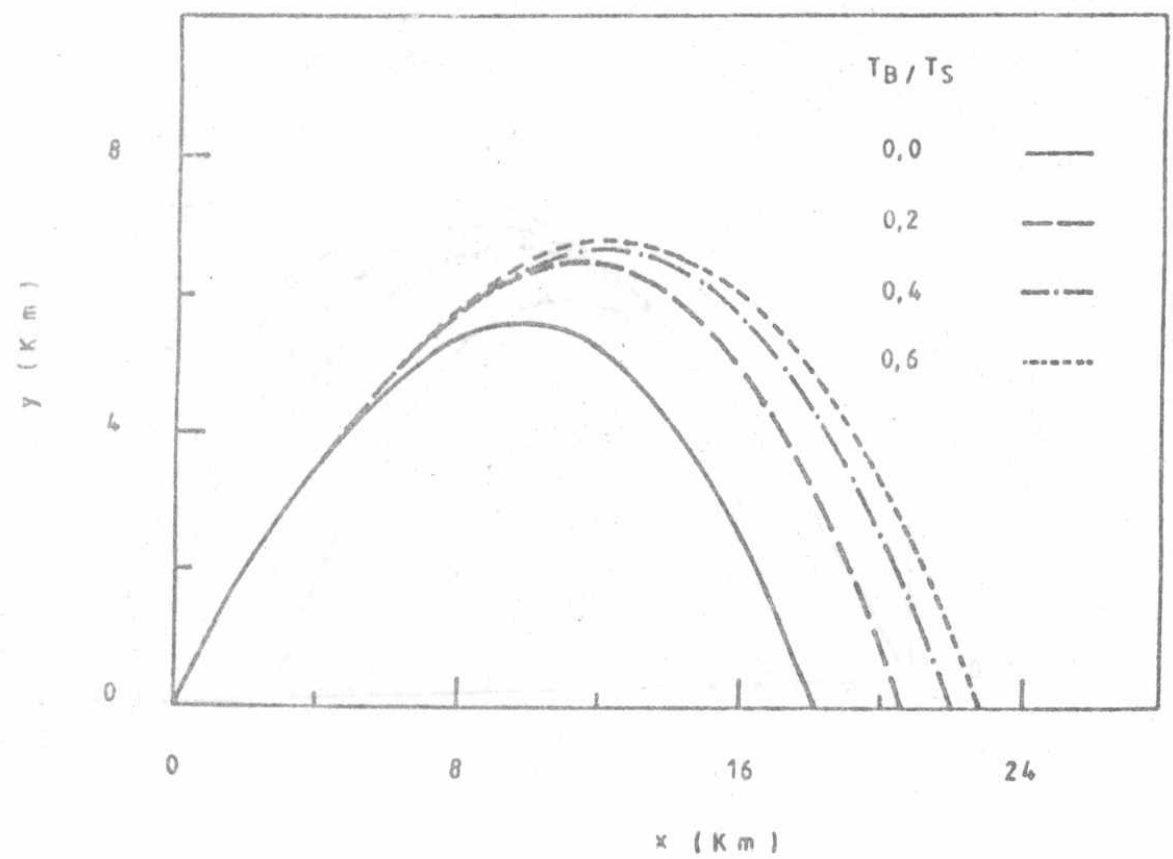

Fig.5. $155 \mathrm{~mm}$ HE Projectile trajectory at different bleeding times for $80 \%$ base drag reduction. 


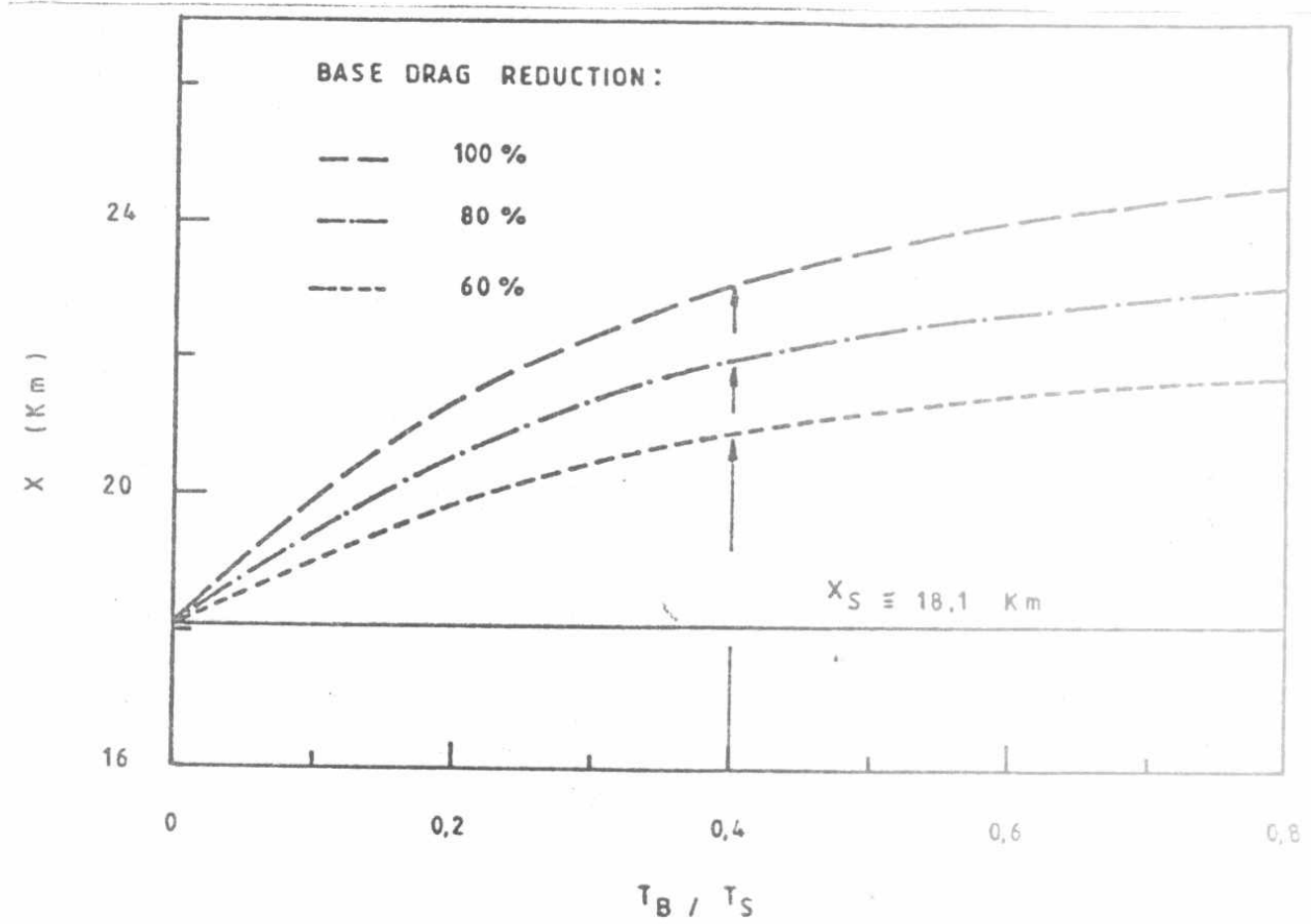

Fig.6. Dependence of maximum range on bleeding time.

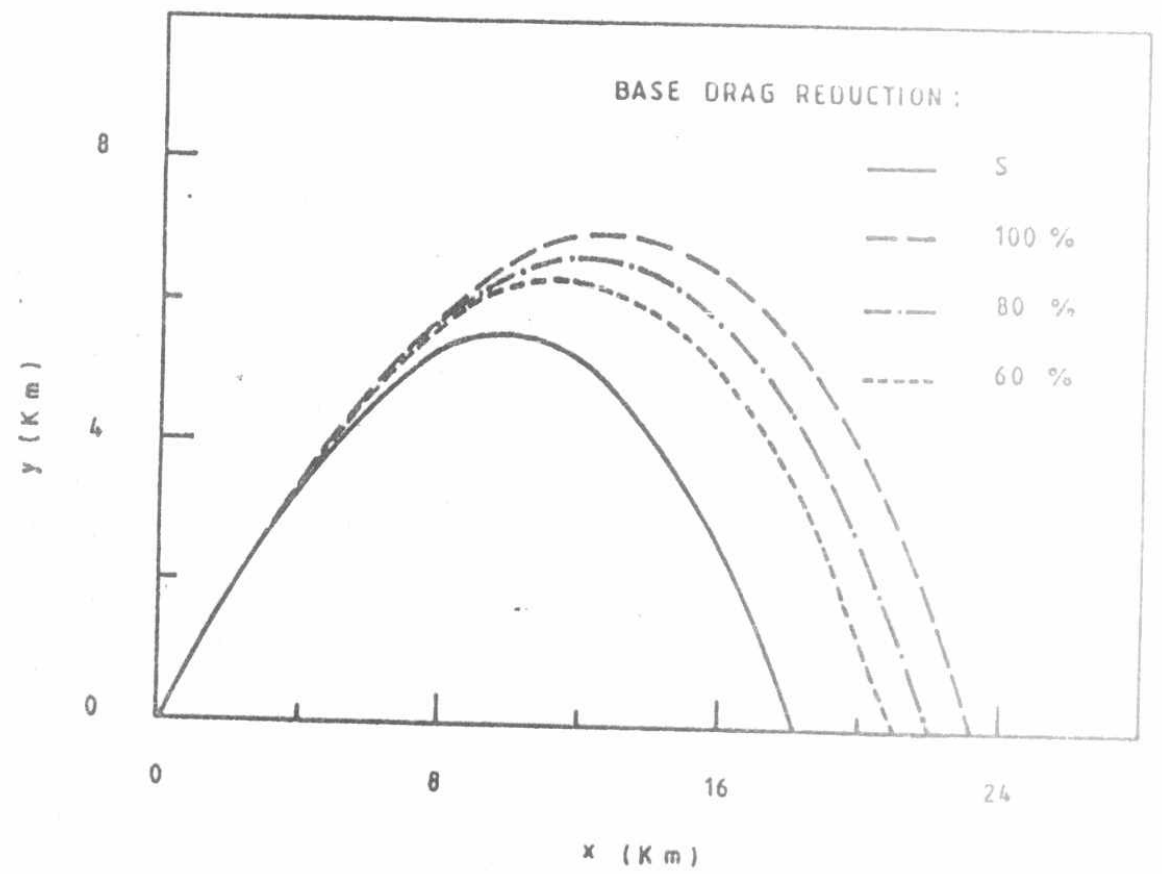

Fig.7. Trajectory of proposed projectile at different base drag reduction ratios for $T_{B} / T_{S}=0.4$. 


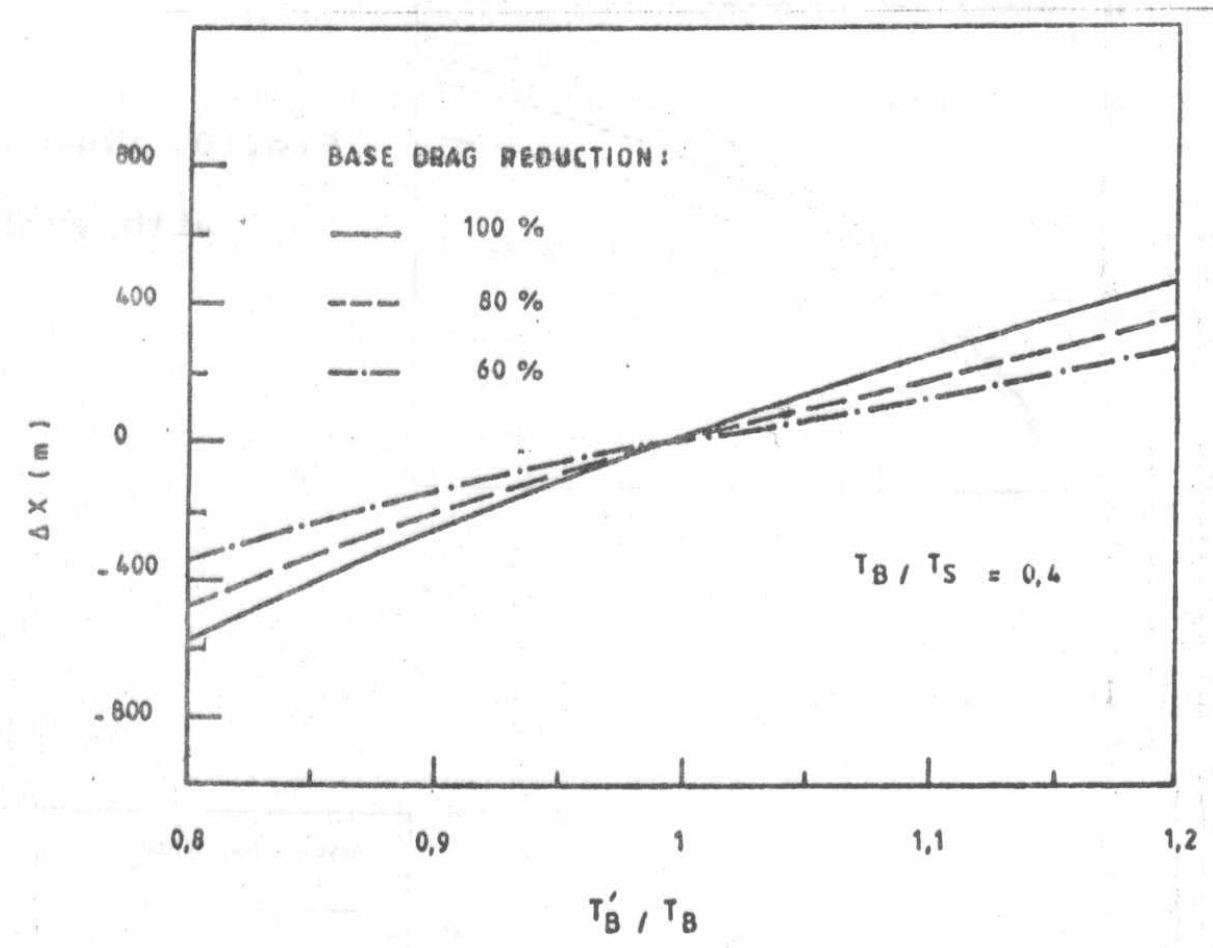

Fig.8. Effect of variation in bleeding time on maximum $r$ ange.

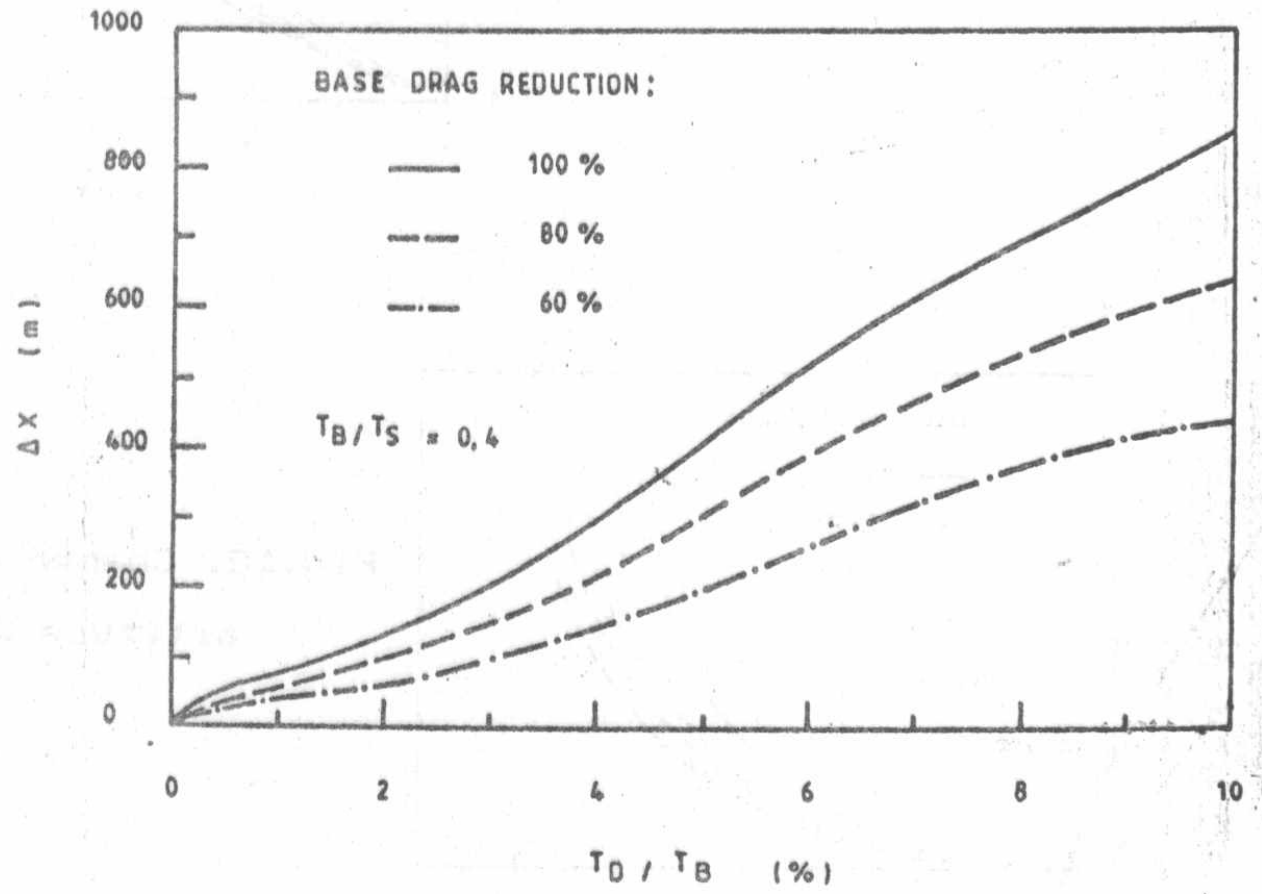

Fig.9. Effect of irregular ignition of base bleed charge on maximum range. 
$\longdiv { M F - 3 \quad 7 6 }$

r
FOURTH ASAT CONFERENCE

14-16 May 1991, CAIRO

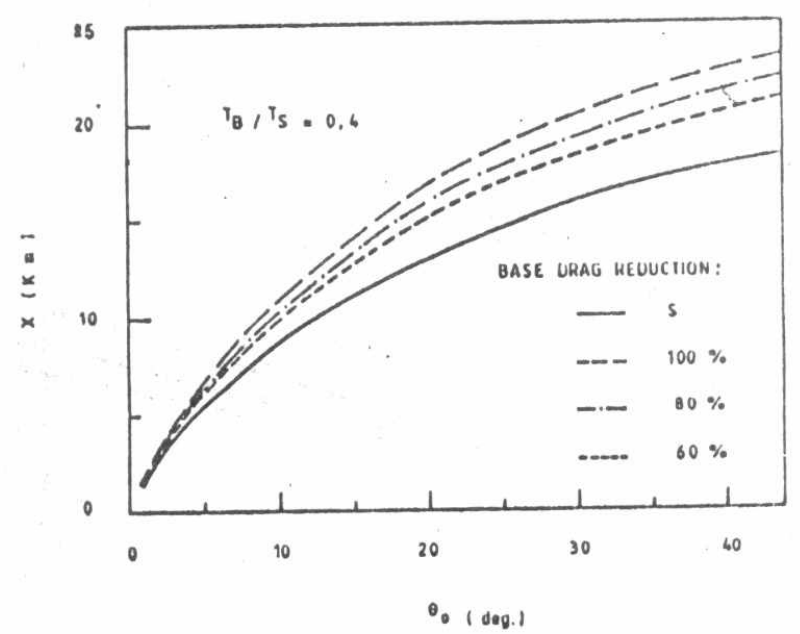

Fig.10. Change of $r$ ange with angle of fire.
Fig.11. Change of flight time with $r$ ange.
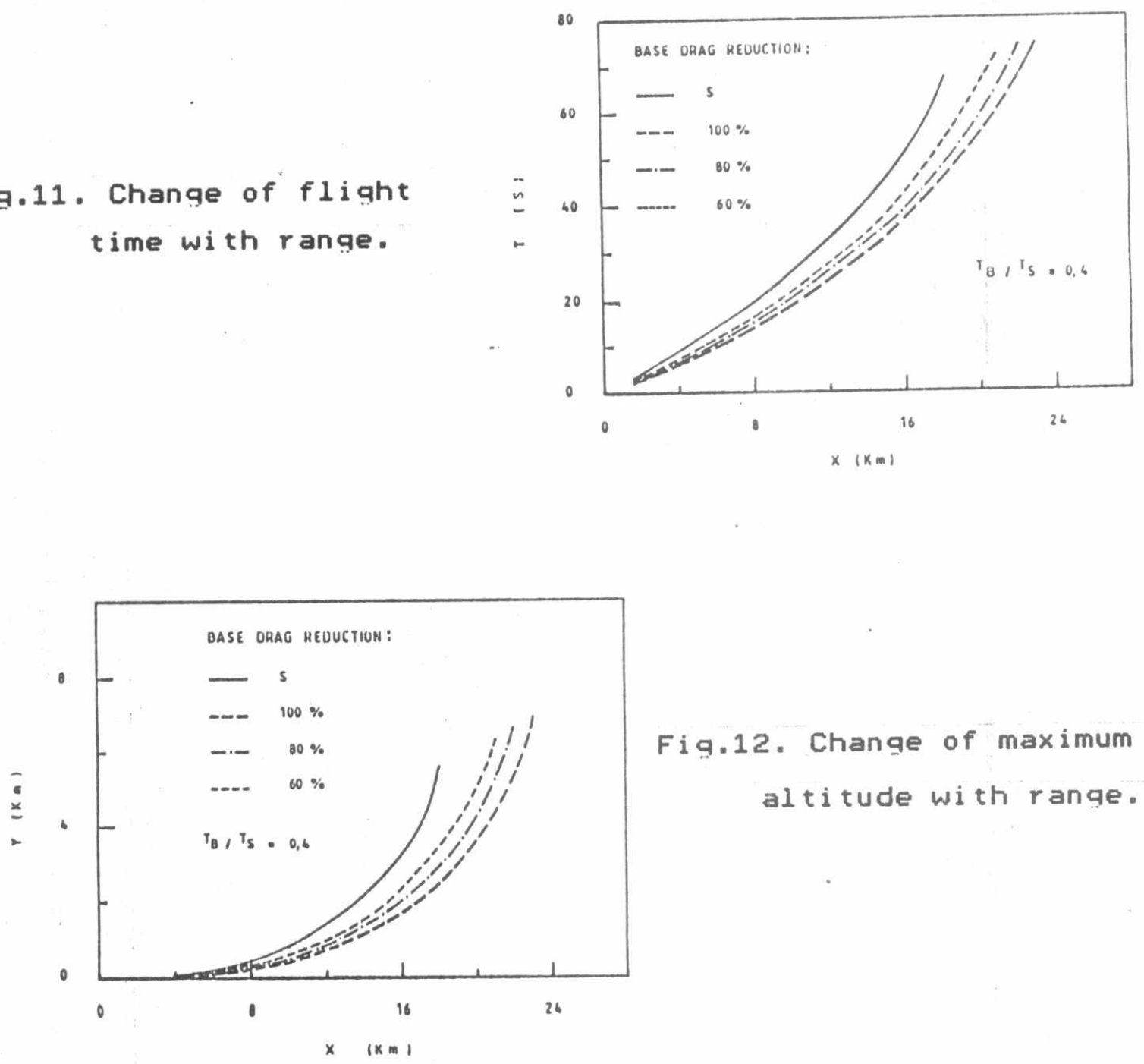

Fig.12. Change of maximum altitude with range. 